\title{
On the spectrum and Martin boundary of homogeneous spaces
}

\author{
S. Northshield \\ Department of Mathematics, SUNY College at Plattsburgh, Plattsburgh, NY 12901, USA \\ Received May 1992; revised January 1994
}

\begin{abstract}
Given a conservative, spatially homogeneous Markov process $X$ on an homogeneous spaces $\mathscr{X}$, we show that if the bottom of the spectrum of the generator of $X$ is zero then the Martin boundary of $\mathscr{X}$ contains a unique point fixed by the isometry group of $\mathscr{X}$.
\end{abstract}

Keywords: Homogeneous space; Markov process; Spectrum; Martin boundary; Fixed point; Amenable group

The motivation for this paper is the study of the connection between the existence of non-constant positive harmonic functions (non-triviality of the Martin boundary) and the existence of non-constant bounded harmonic functions (non-Liouville property) on a Riemannian manifold. This was initially motivated by an analogous problem (Aldous, 1990) for Cayley graphs of finitely generated groups. This paper is an extension of the author's work in that area (see Northshield, 1992). Let $\lambda$ be the spectral radius of the generator $\Delta$ of a conservative and spatially homogeneous Markov process on a homogeneous space $\mathscr{X}$ (for example, $\Delta$ could be the Laplace-Beltrami operator $\Delta$ on a Lie group $\mathscr{X}$ ). We show that $\lambda$ is zero if and only if every superharmonic function is nearly constant on arbitrarily large metric balls. From this, if $\lambda$ is zero, then the Martin boundary has a point fixed by each isometry of $\mathscr{X}$. For a Lie group $\mathscr{X}$, the following chain of implications is known: $\mathscr{X}$ has trivial Martin boundary $\Rightarrow \mathscr{X}$ is Liouville $\Rightarrow \mathscr{X}$ is amenable $\Rightarrow \lambda$ is zero (see Theorem 6). Therefore, if
$\mathscr{X}$ acts transitively on its Martin boundary, then all four conditions are equivalent.

Let $(\mathscr{X}, d)$ be a connected separable metric space with isometry group $\Gamma$ acting transitively on $\mathscr{X}$. Let $\mu$ be a $\Gamma$-invariant measure on the Borel sets of $\mathscr{X}$. We define a (left) action of $\Gamma$ on the space $\mathscr{C}(\mathscr{X})$ of continuous real functions on $\mathscr{X}$ by

$g \cdot F(x)=F\left(g^{-1} x\right), \quad F \in \mathscr{C}(\mathscr{X}), \quad g \in \Gamma$.

Let $X=\left\{X_{t}: t \geqslant 0\right\}$ denote a Markov process on $\mathscr{X}$ and let $\left\{P_{t}: t \geqslant 0\right\}$ denote the corresponding semigroup (see, for example, Ito, 1984). We assume $X$ is conservative (i.e. $P_{t} 1=1$ ) and is spatially homogeneous in the sense that for all $t \geqslant 0, g \in \Gamma$, and $F \in \mathscr{C}(\mathscr{X})$,

$g \cdot\left(P_{t} F\right)=P_{t}(g \cdot F)$.

Further, we assume that there is a $\Gamma$-invariant measure $\mu$ (e.g. Haar measure if $\mathscr{X}$ is itself a group) such that, for $t>0$ and $x \in \mathscr{X}$, the measure $p(t, x, \cdot) \equiv P_{t} X \cdot(x)$ is equivalent to $\mu$. By the Lindelöf property of $\mathscr{X}$, the invariance of $\mu$, and the 
equivalence of $\mu$ with $p(t, x, \cdot)$,

$p(t, x, A)>0$

for every non-empty open set $A$.

Define $p(t, x, y)$ to be the Radon-Nikodym derivative $[\mathrm{d} p(t, x, \cdot) / \mathrm{d} \mu](y)$. Then condition (1) becomes, for $g \in \Gamma, x, y \in \mathscr{X}$, and $t \geqslant 0$,

$p(t, x, y)=p(t, g x, g y)$.

The generator $\Delta$ of $X$ is defined by

$$
\Delta f(x)=\lim _{t \searrow 0}\left[P_{t} f(x)-f(x)\right] / t
$$

and we let $\mathscr{D}(\Delta)$ denote the domain of $\Delta$. Let $\mathscr{S}=\{F \in \mathscr{D}(\Delta): F>0, \Delta F \leqslant 0\}$ be the set of positive superharmonic functions. We assume that

$\log \mathscr{S}=\{\log F: F \in \mathscr{S}\}$ is equicontinuous.

This condition is equivalent to the equicontinuity of $\left\{F \in \mathscr{S}: F\left(x_{0}\right)=1\right\}$ for a (any) point $x_{0}$ and is implied by a strong Harnack inequality (see Loeb and Walsh, 1965). The bottom of the spectrum of $-\Delta$ is given by

$\lambda_{\mathscr{X}}=\sup \{\lambda: \exists f \in \mathscr{D}(\Delta): f>0$ and $\Delta f+\lambda f \leqslant 0\}$.

We define Green's operators by

$G^{\lambda} v(x) \equiv \int_{0}^{\infty} P_{t} v(x) \mathrm{e}^{\lambda t} \mathrm{~d} t$

and we say that $G^{\lambda}$ exists if there exists a positive function $v$ such that $G^{\lambda} v(x)$ exists.

Lemma 1. $\lambda_{x}=\sup \left\{\lambda: G^{\lambda}\right.$ exists $\}$.

Proof. Let $\lambda, t \geqslant 0$, and $f \in \mathscr{D}(\Delta)$. By the semigroup property, if $G^{\lambda} f(x)<\infty$, then

$P_{t} G^{\lambda} f(x)=\mathrm{e}^{-\lambda t} \int_{t}^{\infty} P_{s} f(x) \mathrm{e}^{\lambda s} \mathrm{~d} s$

and therefore,

$\Delta G^{\lambda} f(x)=-\lambda G^{\lambda} f(x)-f(x)$.

Note that by Fatou's lemma, if $f \in \mathscr{S}$, then

$P_{t} \Delta f(x) \geqslant \Delta P_{t} f(x)$ and therefore, by applying Fatou's lemma again, we have

$\int_{0}^{\infty} \mathrm{e}^{\lambda t} \Delta P_{t} f(x) \mathrm{d} t \geqslant \Delta \int_{0}^{\infty} \mathrm{e}^{\lambda t} P_{t} f(x) \mathrm{d} t$.

Let $\lambda<\varepsilon<\lambda_{x}$. By definition, there exists a positive function $f$ in $\mathscr{D}(\Delta)$ such that $\Delta f+$ $\varepsilon f \leqslant 0$. Let $v=-(\Delta f+\lambda f)$. Then $v=-(\Delta f+\varepsilon f)+$ $(\varepsilon-\lambda) f>0$. By (4) and (5), $G^{\lambda} v(x) \leqslant f(x)$, and, since $v>0, G^{\lambda}$ exists.

To show the converse, suppose $f \equiv G^{\lambda} v$ exists for some $v>0$. Then, by (4), $\Delta f(x)+\lambda f(x)=$ $-v(x)<0$ and therefore $\lambda_{x} \geqslant \lambda$.

Lemma 2. The following are equivalent:

(a) $\lambda_{\mathscr{X}}=0$,

(b) for all $f \in \mathscr{S}, \sup _{x} P_{t} f(x) / f(x)=1$.

Proof. (a) $\Rightarrow$ (b). We shall prove the contrapositive. Suppose $f$ is a positive superharmonic function such that $P_{r} f \leqslant c f$ for some $c<1$ and some $r$. Since $f$ is superharmonic, $P_{t} f(x)$ is a decreasing function of $t$ and so, by induction,

$P_{r n} f \leqslant c^{n} f, \quad n=1,2, \ldots$.

and so

$P_{t} f \leqslant c^{\lfloor t r\rfloor} f, \quad t \geqslant 0$.

Recall that $c<1$ and so we can choose $\lambda>0$ so that $c \mathrm{e}^{\lambda r}<1$. Then

$$
\begin{aligned}
& \int_{0}^{\infty} \mathrm{e}^{\lambda t} P_{t} f(x) \mathrm{d} t \\
& \quad=\sum_{n \geqslant 0} \int_{n r}^{n r+r} \mathrm{e}^{\lambda t} P_{t} f(x) \mathrm{d} t \\
& \quad \leqslant f(x) \sum_{n \geqslant 0} c^{n} \int_{n r}^{n r+r} \mathrm{e}^{\lambda t} \mathrm{~d} t \\
& \quad<\infty .
\end{aligned}
$$

Hence, $G^{\lambda}$ exists and, by Lemma $1, \lambda_{x}>0$.

(b) $\Rightarrow$ (a). Suppose $\lambda_{x}>0$. By Lemma 1 , there exists $f \in \mathscr{S}$ and $\lambda>0$ such that $G^{\lambda} f$ exists. By (3), $P_{t} G^{\lambda} f(x)$

$$
\begin{aligned}
& =\mathrm{e}^{-\lambda t} \int_{t}^{\infty} P_{s} f(x) \mathrm{e}^{\lambda s} \mathrm{~d} s \\
& \leqslant \mathrm{e}^{-\lambda t} G^{\lambda} f(x)
\end{aligned}
$$


and so $G^{\lambda} f \in \mathscr{S}$ and $\sup _{x} P_{t} G^{\lambda} f(x) / G^{\lambda} f(x)$ $\leqslant \mathrm{e}^{-\lambda t}<1$.

Lemmas 1 and 2 hold for arbitrary Markov processes. In the next theorem, all of the assumptions above are necessary.

Theorem 3. The following are equivalent:

(a) $\lambda_{x}=0$,

(b) for every $f \in \mathscr{S}$, there exists a sequence $g_{n} \in \Gamma$ such that for every $x, y \in \mathscr{X} f\left(g_{n} x\right) / f\left(g_{n} y\right) \rightarrow 1$,

(c) for all $f \in \mathscr{S}$ and $\varepsilon, r>0$, there exists a ball $B$ of radius $r$ such that

$\sup _{y, z \in B} f(y) / f(z)<1+\varepsilon$

Proof. (a) $\Rightarrow$ (b). Suppose $\lambda_{x}=0$ and $f \in \mathscr{S}$. Then, by Lemma 2 ,

$\sup _{x} P_{t} f(x) / f(x)=1$.

Let $f_{x}(y)=[f(y) / f(x)]^{1 / 2}$. Since, for any $g$, $P_{t} g^{1 / 2} \leqslant\left[P_{t} g\right]^{1 / 2} \leqslant g^{1 / 2}$ (Schwarz inequality), both $f_{x}$ and $f_{x}^{2}$ are positive and superharmonic. Since $P_{t} f_{x}(x)=P_{t}\left(f^{1 / 2}\right)(x) / f^{1 / 2}(x)$

$\sup _{x} P_{t} f_{x}(x)=1$.

Since $1=\left[f_{x}(x)\right]^{2} \geqslant P_{t}\left(f_{x}^{2}\right)(x) \geqslant\left[P_{t} f_{x}(x)\right]^{2}$, there exists a sequence $x(n)$ such that both $P_{t} f_{x(n)}(x(n))$ and $P_{t}\left[f_{x(n)}^{2}\right](x(n))$ converge to 1 as $n \rightarrow \infty$. Choose $g_{n} \in \Gamma$ such that $x_{n}=g_{n} x_{0}$, and define

$F_{n}(x)=\left|\left[f\left(g_{n} x\right) / f\left(g_{n} x_{0}\right)\right]^{1 / 2}-1\right|$.

Then, by (1),

$P_{t} F_{n}^{2}\left(x_{0}\right)=P_{t}\left(\left(f_{x(n)}-1\right)^{2}\right)\left(x_{n}\right) \rightarrow 0$

and therefore, by Jensen's inequality,

$P_{t} F_{n}\left(x_{0}\right) \rightarrow 0$.

Since $\left\{f\left(g_{n} \cdot\right) / f\left(g_{n} x_{0}\right)\right\}$ is equicontinuous (because $\log \mathscr{S}$ is), it follows that $\left\{F_{n}\right\}$ is equicontinuous. By (2), we have that $F_{n} \rightarrow 0$ pointwise.

(b) $\Rightarrow$ (c). Let $f \in \mathscr{S}$ and suppose that there is a sequence $\left(g_{n}\right)$ in $\Gamma$ such that $f\left(g_{n} x\right) / f\left(g_{n} y\right) \rightarrow 1$ for all $x, y \in \mathscr{X}$. Let $\phi_{n}=f\left(g_{n} \cdot\right) / f\left(g_{n} x_{0}\right)$. Since $\phi_{n}$ con- verges to 1 pointwise and since $\left\{\phi_{n}\right\}$ is equicontinuous (because $\phi_{n} \in \mathscr{S}$ and $\log \mathscr{S}$ is equicontinuous), $\phi_{n}$ converges to 1 uniformly on $B\left(x_{0}, r\right)$.

(c) $\Rightarrow$ (a). By condition (c), given $\varepsilon, r>0$, we may choose $x$ such that for all $y, z \in B(x, r)$, $1-\varepsilon \leqslant f(y) / f(z)$. Then

$$
\begin{aligned}
& P_{t} f(x) / f(x) \\
& \quad \geqslant \int_{B(x, r)} p(t, x, \mathrm{~d} z) f(z) / f(x) \\
& \quad \geqslant(1-\varepsilon) p(t, x, B(x, r)) .
\end{aligned}
$$

Since $X$ is conservative and $\varepsilon, r$ are arbitrary, $\sup _{x} P_{t} f(x) / f(x)=1$. By Lemma $2, \lambda_{x}=0$.

We now recall the definition of the Martin boundary. If Green's function $G(x, y)=\int_{0}^{\infty} p(t, x, y)$ $\mathrm{d} t$ does not exist, we say that the Martin boundary is trivial (composed of one point). If Green's function exists, define $K(x, y)=G(x, y) / G\left(x, x_{0}\right)$. Clearly $K(\cdot, y)$ is super-harmonic for all $y \in \mathscr{X}$. Let $\Phi$ be the set of all sequences $\alpha(n)$ such that $K(\cdot, \alpha(n))$ converges pointwise. Given $\alpha, \beta \in \Phi$, we say that $\alpha$ and $\beta$ are equivalent $(\alpha \sim \beta)$ if $K(\cdot, \alpha(n))$ and $K(\cdot, \beta(n))$ have the same pointwise limit. The Martin boundary is the quotient space $M=\Phi / \sim$.

By (1), $G(x, y)=G(g x, g y)$ for all $x, y \in \mathscr{X}$ and $g \in \Gamma$. Hence,

$K(x, g y)=K\left(g^{-1} x, y\right) / K\left(g^{-1} x_{0}, y\right)$

Given $\xi \in M, g \in \Gamma$, and any sequence $y_{n} \rightarrow \xi$, the sequence $K\left(\cdot, g y_{n}\right)$ is in $\mathscr{S}$. Furthermore, for any other sequence $z_{n} \rightarrow \xi, K\left(\cdot, g z_{n}\right)$ and $K\left(\cdot, g y_{n}\right)$ converge to the same limit. We then define $g \xi$ to be that element of $M$ corresponding to this limit and thus have an action of $\Gamma$ on $M$ defined by

$K(x, g \xi)=K\left(g^{-1} x, \xi\right) / K\left(g^{-1} x_{0}, \xi\right)$.

A positive real-valued continuous function on the $\Gamma$-space $\mathscr{X}$ is said to be a character if for all $x, y \in \mathscr{X}$ and $g \in \Gamma$,

$F(g x) / F(x)=F(g y) / F(y)$.

Lemma 4. If $\lambda_{x}=0$, then every superharmonic character is constant. 
Proof. Let $F$ be a superharmonic character, fix $x_{0} \in \mathscr{X}$, and assume, without loss of generality, that $F\left(x_{0}\right)=1$. Define $\chi(g)=F(g x) / F(x)$ for some (any) $x \in \mathscr{X}$. By $(8)$,

$g \cdot F=\chi\left(g^{-1}\right) F$.

Then, by (1),

$P_{t} F(g x)$

$$
=g^{-1} \cdot P_{t} F(x)=P_{t}\left(g^{-1} \cdot F\right)(x)=\chi(g) P_{t} F(x)
$$

and so, for all $g, x$, and $t$,

$P_{t} F(x) / F(x)=P_{t} F(g x) / F(g x)$.

By transitivity, for some function $\alpha$,

$P_{t} F(x)=\alpha(t) F(x)$.

By the semigroup property, $\alpha(t+s)=\alpha(t) \alpha(s)$ and, since $\alpha$ is continuous (because $F \in \mathscr{D}(\Delta)$ ), $\alpha(t)=\mathrm{e}^{k t}$ for some $k$. Therefore,

$P_{t} F=\mathrm{e}^{k t} F$.

Since $F$ is superharmonic, $k \leqslant 0$ and, since $\lambda_{x}=0, k \geqslant 0$. Therefore, $P_{t} F=F$. Since $F^{1 / 2}$ is also a superharmonic character, it follows that the variance of $F\left(X_{t}\right)^{1 / 2}$ is zero and therefore, by (2), $F$ is constant.

Theorem 5. If $\lambda_{x}=0$, then there exists a unique point in the Martin boundary fixed by $\Gamma$.

Proof. Suppose $\lambda_{x}=0$. If Green's function does not exist, then the Martin boundary is trivial. Suppose $G$ exits. By Theorem 3 , since $K\left(\cdot, x_{0}\right)$ is superharmonic, there exists a sequence $g_{n} \in \Gamma$ such that

$K\left(g_{n} x, x_{0}\right) / K\left(g_{n} x_{0}, x_{0}\right) \rightarrow 1$.

By (6),

$K\left(x, g_{n}^{-1} x_{0}\right) \rightarrow 1$.

Hence $g_{n}^{-1} x_{0}$ converges to some $\xi_{0} \in M$ and $K\left(\cdot, \xi_{0}\right) \equiv 1$. By (7), since $K\left(\cdot, g \xi_{0}\right)=1$ and thus $g \xi_{0}=\xi_{0}$ for any $g, \xi_{0}$ is a fixed point.

To show uniqueness, let $\xi$ be a fixed point of the Martin boundary. Since $K(\cdot, \xi)=K\left(\cdot, g^{-1} \xi\right)$, we have, by (7),

$K(x, \xi)=K(g x, \xi) / K\left(g x_{0}, \xi\right)$ and therefore $K(\cdot, \xi)$ is a character. Since $K(\cdot, \xi)$ is a limit of superharmonic functions, $K(\cdot, \xi)$ is itself superharmonic. By Lemma $4, K(\cdot, \xi)$ is constant and so identically 1 . Hence $\xi=\xi_{0}$.

As an application, we restrict ourselves to the case where $\mathscr{X}$ is a Lie group and $X_{t}$ is Brownian motion on $\mathscr{X}$ (generated by the Laplace-Berltrami operator 4 ). We say that $\mathscr{X}$ is Liouville if there are no non-constant bounded harmonic functions on $\mathscr{X}$.

Theorem 6. If $\mathscr{X}$ is a Lie group which acts transitively on its Martin boundary then the following conditons are equivalent.

(a) $\lambda_{x}=0$,

(b) the Martin boundary is trivial,

(c) $\mathscr{X}$ is Liouville,

(d) $\mathscr{X}$ is amenable.

Proof. (a) $\Rightarrow$ (b). This follows immediately from Theorem 5.

(b) $\Rightarrow$ (c). This is immediate from the Martin representation theorem (see, for example, Brelot, 1971) and the consequence that if $\mathscr{X}$ has a trivial Martin boundary, then there are no non-constant positive harmonic functions on $\mathscr{X}$.

(c) $\Rightarrow$ (d). As in the proof of Theorem 3 of Lyons and Sullivan (1984), each operator $P_{t}$ commutes with each invariant mean $\psi$ on the amenable semigroup $\mathbb{R}^{+}$and therefore, for any measurable $A$, $\mu(A)(x) \equiv \psi\left(P \cdot 1_{A}(x)\right)$ is bounded harmonic. By (1), $P_{t} 1_{x A}(e)=P_{t} 1_{A}\left(x^{-1}\right)$ and therefore, by the Liouville property, $\mu(x A)(e)=\mu(A)\left(x^{-1}\right)=\mu(A)(e)$. Hence $\mu(\cdot)(e)$ is an invariant mean on $\mathscr{X}$ and $\mathscr{X}$ is amenable.

(d) $\Rightarrow$ (a). Let $C=B^{-}(e, \varepsilon)$. For any compact $K$, let $f$ be a smooth function with range $[0,1]$ (hence in $\mathscr{D}(\Delta)$ ) such that $f$ is 1 on $K, 0$ outside of $C K$, and $|\Delta f| \leqslant c$ (for some $c$ depending on $C$ ). Then $\langle f,-\Delta f\rangle \leqslant c \lambda(C K \backslash K)$ and $\langle f, f\rangle \geqslant \lambda(K)$ where $\langle\cdot, \cdot\rangle$ is the $L_{2}$ inner product with respect to Haar measure $\lambda$ on $\mathscr{X}$. By amenability, $K$ can be chosen so that $\lambda(C K) \backslash K) / \lambda(K)$ is arbitrarily small (see Paterson, 1988, Theorem 4.13). Hence $\langle f,-\Delta f\rangle \mid\langle f, f\rangle$ is arbitrarily small. By Rayleigh's theorem (see Chavel, 1984), the bottom of 
the $L^{2}$ spectrum is 0 which is equivalent, by Theorem 1 of Fischer-Colbrie and Schoen (1980), to $\lambda_{\mathscr{X}}=0$.

\section{References}

Aldous, D. (1990), personal communication.

Brelot, M. (1971), On Topologies and Boundaries in Potential Theory (Springer, Berlin).

Chavel, I. (1984), Eigenvalues in Riemannian Geometry (Academic Press, New York).
Fischer-Colbrie, D. and R. Schoen (1980), The structure of complete stable minimal surfaces in 3-manifolds of non-negative scalar curvature, Comm. Pure Appl. Math. 33, 199-211.

Ito, K. (1984), Lectures on Stochastic Processes (Springer, Berlin).

Loeb, P. and B. Walsh (1965), The equivalence of Harnack's principle and Harmack's inequality in the axiomatic system of Brelot, Ann. Inst. Fourier (Grenable) 15(2), 597-600.

Lyons, T. and D. Sullivan (1984), Function theory, random paths and covering spaces, J. Differential Geom. 19, 299-323.

Northshield, S. (1992), Amenability and superharmonic functions, Proc. Amer. Math. Soc., 119(2), 561-566.

Peterson, A.L.T. (1988), Amenability (AMS, Providence). 